\title{
Method of Elaboration of Detailed Existing Land Use (ELU) Map of Poland
}

\author{
Dariusz Dukaczewski ${ }^{\mathrm{a}}$ * \\ ${ }^{a}$ IGiK - Institute of Geodesy and Cartography, 27 Modzelewskiego St. 02-679 Warsaw, Poland, dariusz.dukaczewski@igik.edu.pl \\ * Corresponding author
}

Keywords: Detailed Existing Land Use Map, Remote Sensing Data, GIS Analysis

\begin{abstract}
:
During few last years in many European Union countries the growing interest in land use monitoring of the level of details corresponding to the Urban Atlas or CORINE Land Cover level 6 (1: 10000 scale) was observed. This kind of information is very useful for detailed spatial analyses of multifunctional urbanized and/or industrialized areas i.e. for spatial planning. The advent of new, very high resolution satellite data, lidar and UAV multispectral data together with new possibilities of analyses allow to achieve rich, up-to-date spatial information on Existing Land Use (ELU). This kind of information is of great economic significance for public administration of local, regional and central level, as a basis for land use/land cover monitoring and analyses concerning Planned Land Use (PLU). It should be emphasized that public administration in Poland can employ the topographic database (BDOT10k) of level of details corresponding to the 1: 10000 scale map for part of these analyses. However, it should be stressed that thematic scope of spatial information needed by public administration is in significant part more detailed or different of this, which is available in the case of topographic database (i.e. information on health and sanitary state of the forests, more in-depth classification of meadows and pastures, areas of natural succession). This way the public administration needs both detailed topographic database and detailed existing land use map, which should be wholly interoperable. The ELU map should also be harmonized with Hierarchical INSPIRE Land Use Classification System (HILUCS). Till now the Polish public administration has access to CORINE Land Cover level 3 data of 1990, 2000, 2006, 2012, 2018 (for the whole territory), 1975 and 1884/1885 CORINE database of Odra river basin, CORINE Land Cover level 4 experimental database of 1995 (for fragments of south-west and south part of Poland), Soil Sealing and HRL databases (for the whole area), Urban Atlas for big cities, as well as Land Use Map of Poland of 1935 - 1939 (published in 1969), Land Use Map of Poland of 1977-1978 and many detailed, but local land use maps of 1916 - 2015 period. This data doesn't meet the recent needs of public administration. To meet the formulated requirements it was necessary to propose the CORINE Land Cover level 6 \& 5 nomenclature (harmonized in thematic scope with BDOT10k topographic database and HILUCS nomenclature), to elaborate and test the method of building and updating the databases (employing the remote sensing and in-situ data) and to formulate the guidelines. It was also necessary to analyse and to synthesise the possibilities and limitations of use an information from Polish old land use and city maps containing detailed land use information, which should be useful for detection of long-term land use / land cover changes for areas of complex functions and relatively fragile ecosystems.
\end{abstract}

In the research reported here, the author proposed the CORINE Land Cover level 6 \& 5 nomenclature of Poland ${ }^{1}$ in conformity with public administration requirements, and in accordance with CORINE Land Cover level 4 'Smolenice' legend (Feranec, Ot’ahel’, 1998; European Landscape Dynamics: CORINE Land Cover Data, 2016). The classes of proposed nomenclature were harmonized with types of objects of national topographic database of Poland (BDOT10k) and HILUCS nomenclature. The author analysed, tested and synthesised the possibilities of building the CORINE Land Cover level 4, 5 \& 6 databases, employing the Copernicus project Sentinel-2 and commercial WorldView-3 \& 4 satellite data, as well as reclassified BDOT10k database data. The results of carried tests have demonstrated that Sentinel-2 data (together with BDOT10k data) can be used for mapping of 94,3 \% territory of Poland (294 $\left.856 \mathrm{~km}^{2}\right)$, while for $5,7 \%\left(17823 \mathrm{~km}^{2}\right)$ the satellite data of better spatial resolution and BDOT10k data are necessary. The tests have revealed that in the case of CORINE Land Cover level 4 about $12 \%$ of classes can be classified automatically, $16 \%$ - semi-automatically and $72 \%$ - visually (on screen). In the case of CLC level 5 only $8 \%$ of classes can be classified automatically, $5 \%$ - semi-automatically, but $89 \%$ - visually. Almost $97 \%$ of CLC level 6 classes can be classified visually, $2 \%$ - automatically and only $1 \%$ - semi-automatically. These statistics have demonstrated the need to apply two methods of building of ELU maps of Poland - 'from general to detailed information' (1) and 'from detailed to general information' (2). The first method is applicable when building the CLC level 4 and level 5 database, while second - in the case of CLC level 6 database. In the case of first method the main source is remote sensing data from Sentinel-2, WorldView-3 \& 4, and auxiliary data is BDOT10k. The first stage is verification of most recent CLC

\footnotetext{
${ }^{1}$ Proposed CLC level 5 and 6 nomenclature of Poland is available at: http://www.igik.edu.pl/upload/File/drdd/2016_CORINE_5_6.pdf
} 
level 3 database. The second stage is an automatic classification of selected classes of CLC level 4. The resulting masks, together with aggregated BDOT10k types of objects are employed to reclassify CLC level 3 database into the CLC level 4 database. The similar solution is applied in the case of building of CLC level 5 database. In the case of CLC level 6 database the considerable number of classes is similar to the BDOT10k types of objects and/or groups of objects. This way the first stage of second method is BDOT10k types of objects aggregation. The resulting CLC level 6 classes polygons are compared (and adjusted if necessary) with related CLC level 5 and Urban Atlas polygons. Then, employing the Sentinel-2 and WorldView-3 \& 4 data, as well as available in-situ thematic databases the others CLC level 5 polygons are reclassified (or geometrically adjusted) into the CLC level 6 polygons database.

Due to the need of updating of the ELU map in 4 -year cycle it was necessary to propose the procedure of land use / land cover changes detection, employing NDVI, SAVI and TSAVI indexes.

To facilitate the multitemporal analyses and detection of long-term land use / land cover changes, it was necessary to carry out an analysis of possibilities and limitations of use an information from Polish old land use and city maps containing detailed land use information, as well as from topographic maps of 1606 - 1939 period (Dukaczewski, Bielecka, 2017). The result of analysis of 2010 maps was the metadata database, including an information on number of land use / land cover classes, corresponding to the CLC level 4, 5, 6, Urban Atlas, HILUCS and types of objects of BDOT10k, respecting the Data Specification on Land Cover - Technical Guidelines (2013) schema. Proposed metadata database as well as metadata query tool are adapted for usage of Polish CLC level 5 and 6, as well as national topographic database codes, but it should be stressed that it is possible to take also into the consideration the codes employed in other CLC 5 and 6 national nomenclature and other national topographic databases. This can allow to use the proposed conception of metadata database and metadata query tool also in other countries.

D2.8.II.2. Data Specification on Land Cover - Technical Guidelines (2013) INSPIRE Thematic Working Group Land Cover. European Commission Joint Research Centre, Ispra;

Dukaczewski, D., Bielecka, E., 2017, Utility of Old Polish City Maps and Old Land Use Maps for Multitemporal Detailed Land Use Survey, Proceedings, 7th International Conference on Cartography and GIS, 18-23 June 2018, Sozopol, Bulgaria, ISSN: 1314-0604, Eds: Bandrova T., Konečný M.: 16 - 25;

Feranec, J., Ot'ahel', J., 1998, Final version of the $4^{\text {th }}$ level CORINE land cover classes at scale 1: 50000 (Task 4.2 ). Technical Report, EEA Phare Topic Link on Land Cover, Bratislava, Institute of Geography, SAS;

European Landscape Dynamics: CORINE Land Cover Data, 2016, Eds: Jan Feranec, Tomas Soukup, Gerard Hazeu, Gabriel Jaffrain, CRC Press, Taylor \& Francis Group. 